\title{
Zwei Lösungsansätze zum Brachlandproblem im Val d'Anniviers
}

In den sechziger Jahren erfolgte ein schlagartiger Exodus der Bevölkerung aus dem Val d'Anniviers in den Raum des Wachstumspoles Sierre. Konsequenzen waren in den Bergdörfern ein Rückgang der Landwirtschaftsbetriebe, besonders der hauptberuflichen Betriebe, und eine Reduktion der bewirtschafteten Landflächen. Zusätzlich zu den Direktzahlungen an die Landwirtschaft kamen im Val d'Anniviers folgende Problemlösungsansätze zur Anwendung:

- primär für produktive Landwirtschaft, sekundär für Landschaftspflege:

Einzelbetriebssanierungen z. B. in St. Luc (BUGMANN 1973).

Errichtung von Gemeinschaftsställen in Ayer, Grimentz, Chandolin, St. Jean.

- im Dienste der Landschaftspflege für den Tourismus:

Aufnahme eines Feuerschutzparagraphen in das Polizeireglement der Gemeinde St.Luc im Jahre 1972 (BUGMANN 1974).

Weidenutzung für Jungvieh aus dem Unterland, beschrieben bei BUGManN $(1976,1980)$ als Modell St. Luc.

Nebenberufliche Genossenschaft mit Gemeinschaftsstall als Modell Vissoie, beschrieben bei BUGMANN (1980).

\section{Sozio-ökonomische Vorbedingungen, Organisations- struktur und Wirtschaftlichkeit der Modelle Vissoie und St. Luc}

\section{Das Modell Vissoie}

1973 gab der letzte hauptberufliche Landwirt seinen Betrieb auf. Im Interesse der Aufrechterhaltung der Landschaftspflege bildete sich 1975 eine Genossenschaft von Berufsleuten, die aus landwirtschaftlichen Familien stammten. Jeder warf eine bis mehrere Kühe ins Genossenschaftskapital ein und verpflichtete sich zum Einbringen eines Anteiles an Futter. Ein hauptamtlicher Angestellter hat die Tiere im Gemeinschaftsstall zu besorgen. Die originelle Unternehmung hat 7 Betriebsjahre überdauert und zählt heute 15 Genossenschafter.

Sie schafft 1 Manneinkommen. Je nach Interpretation der nicht sehr durchsichtigen Buchhaltungsabschlüsse wird pro Jahr und eingworfene Kuh ein Ertrag zwischen $+3000 \mathrm{Fr}$. und $-1000 \mathrm{Fr}$. erzielt. Die beim Einbringen von Gras und $\mathrm{Heu}$ an der frischen Luft erworbene Fitneß und die gesellschaftliche Komponente lassen betriebswirtschaftliche Überlegungen offensichtlich zurücktreten. Das sportliche Fieber vor, während und nach Eringerkuh-Kämpfen und der Nervenkitzel beim Handel mit «Reines» sind im Jahresablauf des Dorfgeschehens Höhepunkte. Bemerkenswert ist, dass mit dieser Lösung nicht nur eine Landschaftspflege möglich ist, sondern daß 16 Familien im Dorf mit dem Boden, mit der ursprünglichen Wirtschaftsform und der Tradition verbunden bleiben. Die maßgeblichen Mitglieder der Genossenschaft weisen denn auch mit Nachdruck darauf hin, ihr Unternehmen hätte eine dreifache Bedeutung, nämlich eine ökonomische, eine landschaftsökologische und eine soziologische.

\section{Das Modell St. Luc}

1974 bewirtschafteten 3 hauptberufliche Landwirtschaftsbetriebe rund hundert Hektaren im Dorfgebiet, einen beträchtlichen Teil dieser Fläche mit extensiver Weidenutzung. Über 100 ha wurden nicht bewirtschaftet. Bei diesen Flächen handelte es sich vor allem um nicht maschinell mähbare Wiesen in Steillagen und terrassierte frühere Roggenzelgen.

$\mathrm{Ab} 1975$ wurden diese Flächen eingezäunt und von 120 Haupt Pensions-Jungvieh aus den Kantonen Waadt und Fribourg im Sommer beweidet. Die Gemeinde engagierte sich in dieser Sache im Interesse der Landschaftspflege für den Tourismus.

Auf den Sommer 1983 drängte sich eine Neuorganisation auf, bedingt durch folgende Fakten:

- Einer der drei Landwirtschaftsbetriebe war Ende der siebziger Jahre ausgelaufen, die privat bewirtschaftete Fläche sank auf rund 50 ha.

- Bisher in St. Luc gealpte Kühe aus Vissoie wurden nach Grimentz verlegt; auch Alpungsrechte aus Ayer wurden nach dem Neubau der Alp Nava (Gemeindegebiet Ayer) nicht mehr benützt. Damit sanken die Alpungseinheiten von 120 auf 50 Stösse.

- Die Gemeinde St.Luc suchte die Betreuung des Pensionsviehs aus personellen Gründen zu privatisieren.

Prof. Dr. E. Bugmann, Forschungsstelle für Wirtschaftsgeographie und Raumplanung, Guisanstr. 70, 9010 St. Gallen 


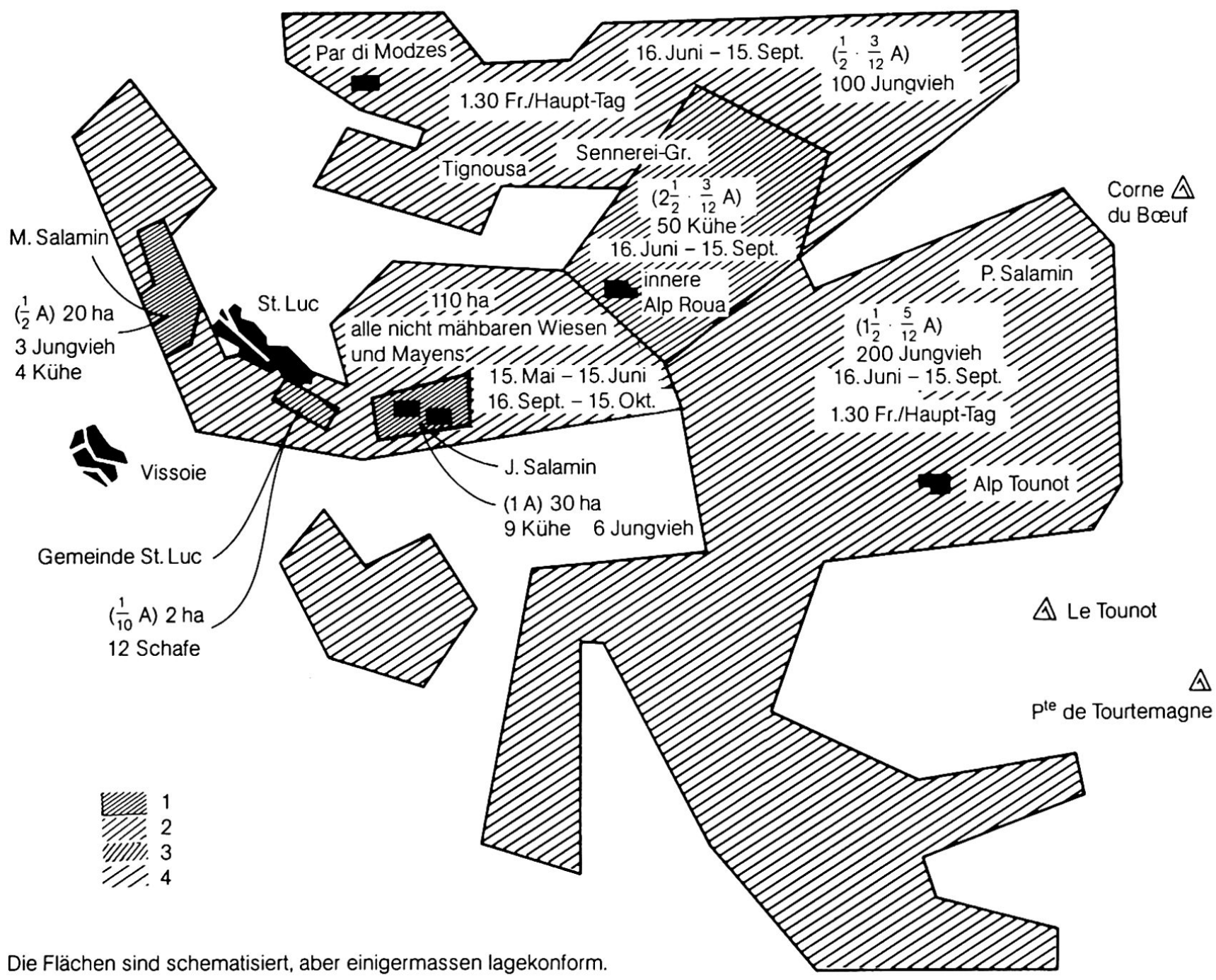

1 Intensive Graswirtschaft, z. T. mit Bewässerung; Weideflächen in Steillagen

2 Innere Alp Roua: Sömmerung von Eringervieh

3 nicht mähbare Wiesen, Mayens und Alpflächen, beweidet durch Unterlandvieh

4 kommunale Schafweide

Die Haltung des Unterland-Jungviehs wurde nun auf den Sommer 1983 durch Vater und Sohn P. Salamin übernommen und im Einvernehmen mit Gemeinde, Alpkorporationen und Landbesitzern wie folgt neu strukturiert (Graphik 1):

1. Die eingezäunte Fläche wird im Dorfgebiet auf 110 ha erweitert. Die Besitzer größerer Landflächen und die Gemeinde erhalten Pachtentschädigungen von insgesamt Fr. 1600.-.

2. Die Jungviehweide wird auf die Alp Tounot ausgedehnt gegen eine Benützungsgebühr von Fr. 5000.- pro Jahr.

3. Auf diesen Flächen können 200 Haupt Jungvieh vom 15. Juni bis 15 . Oktober gehalten werden. Die Pensionsbeiträge belaufen sich für 150 Tage auf
Fr. $39000 .-$, die Sömmerungsbeiträge des Bundes (Fr. 20.- pro Haupt und Tag) auf Fr. 3000.-

Den Einnahmen von Fr. 42 000.- stehen Ausgaben für Pacht und Gebühren sowie Material und Risiko von rund Fr. 9000.- gegenüber. Der Vater wird während der Sommerkampagne halbberuflich, der Sohn vollberuflich bei der lokalen Sesselbahnunternehmung arbeiten, so daß der Saldo von Fr. 33 000.- 7,5 Monatslöhnen entspricht. Somit kann ein Brutto-Monatslohn von Fr. 4400.- erreicht werden.

Die Alpkorporation Roua wird die Alpung der 50 einheimischen Kühe auf die innere Alp Roua konzentrieren, während auf der äußeren Alp (Par di Modzes und Tignousa) für 3 Monate 100 Haupt UnterlandJungvieh in Pension genommen und von der Sennerei-Gruppe betreut werden. Der dabei erzielbare Monatslohn liegt bei etwa Fr. 4500.-. 


\section{Beurteilung und Prognosen für die Zukunft}

- Beide Modelle stellen eine ausreichende Landschaftspflege für den Tourismus sicher.

- Das Modell St. Luc ist in der Sommersaison wegen der großen Zahl weidender Kühe (in Dorfnähe und auf den Alpen) sehr touristenattraktiv.

- Das Modell St. Luc stellt insgesamt 3 landwirtschaftliche Arbeitsplätze, welche auf 8 Arbeitskräfte verteilt und mit andern Beschäftigungen saisonal kombiniert sind, insbesondere mit Anstellungen im Wintertourismus. Bei einem möglichen Auslaufen eines oder beider Individualbetriebe wäre eine Angliederung intensiver Graswirtschaft auf den durch diese Betriebe bewirtschafteten, maschinell mähbaren Flächen möglich.

- Das Modell Vissoie ist offensichtlich ökonomisch wenig effizient, bindet aber mehr Familien direkt an die landwirtschaftliche Arbeit.

- Es ist weiter zu bedenken, daß praktisch jede Bürgerfamilie des Val d'Anniviers Weinberge und Obstgärten am Talhang des Rhonetales nördlich Sierre bewirtschaftet (z. B. in Muraz und Villa). Mit dieser Bindung an die edelste und attraktivste landwirtschaftliche Kulturform wird sich auch die Bereitschaft zu extensiveren Formen der Bodenbewirtschaftung erhalten, insbesondere dann, wenn bereits ein Kern dörflicher Familien mit ihnen beschäftigt sind.
In den beiden hier vorgestellten Fällen haben wesentlich touristische Interessen Anstöße zur Verhinderung der Landschaftsverbuschung gegeben. Im Beispiel Vissoie zeigt sich die positive Wirkung idealistischen Engagements, im Beispiel St.Luc der Stimulans im Hinblick auf die Möglichkeit der Erzielung relativ attraktiver Einkommen.

Die Berggebietsproblematik allgemein und das Brachlandproblem im speziellen wären lösbar, wenn die einheimische Bevölkerung einen in der Tradition verwurzelten Idealismus dank attraktiven Einkommen entfalten könnte, mit anderen Worten, wenn ihr eine Besinnung auf die kulturelle Identität, befreit von der Last wirtschaftlicher Benachteiligung, möglich wäre.

\section{Literatur:}

BUGMANN, E. (1974): St.Luc: Ein Walliser Bergdorf wird Touristenstation. In: Geographica Helvetica Nr. 29, 83-95.

BUGMANN, E. (1976): Neuere Entwicklungen der landwirtschaftlichen Nutzung im Walliser Berggebiet. In: Geographica Helvetica Nr. 31, 65-68.

BUGMANN, E. (1980): Part-time farming and ecological aspects in the Swiss Alps. In: Müller F. e.a.: Geography in Switzerland, Bern, 125-133.

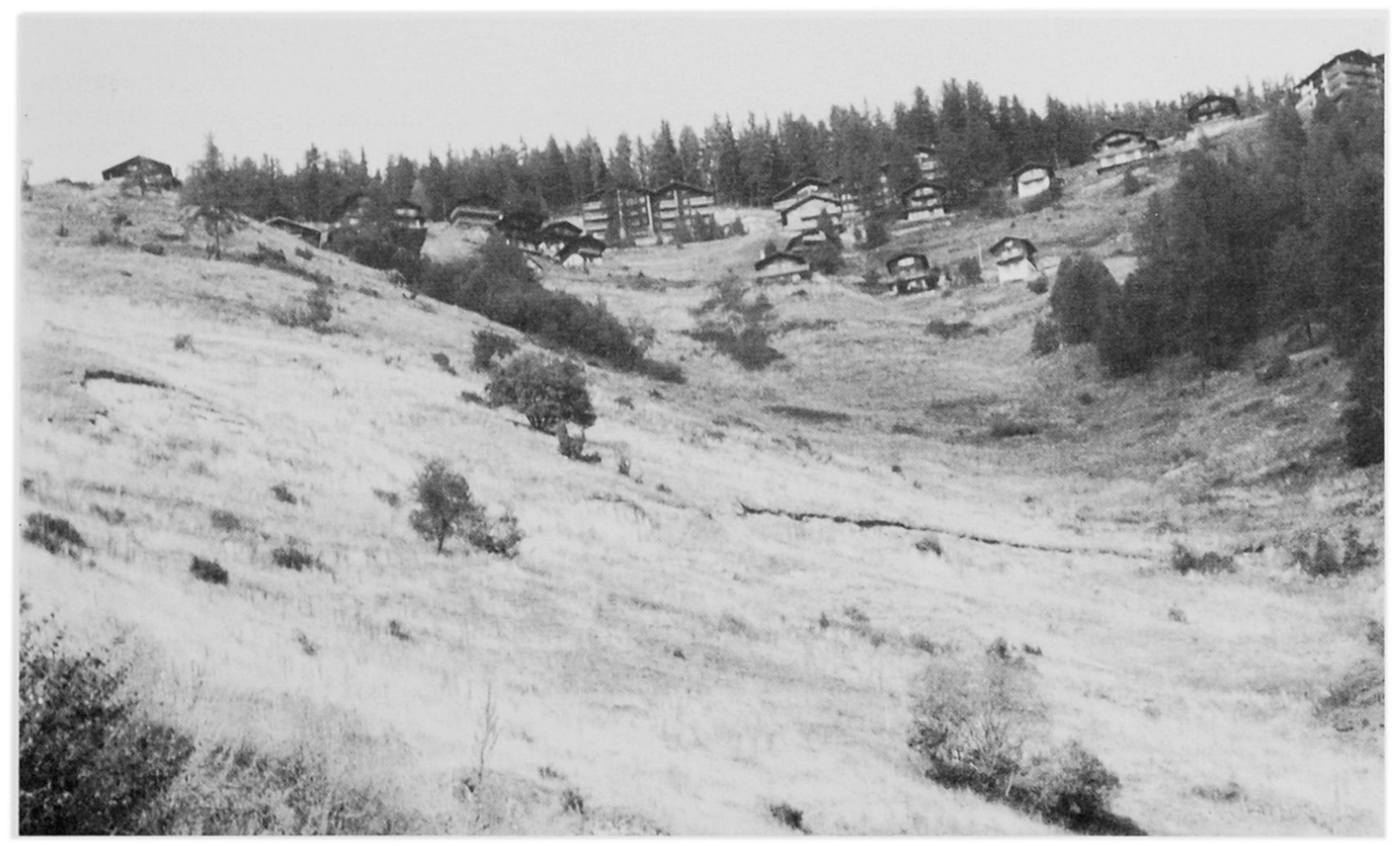

Noch ungelöstes Brachlandproblem in Chandolin, Val d'Anniviers (Aufnahme St. Julen). 